Review

\title{
Electroencephalography (EEG) Technology Applications and Available Devices
}

\author{
Mahsa Soufineyestani ${ }^{\mathbb{D}}$, Dale Dowling and Arshia Khan * $\mathbb{D}$ \\ Department of Computer Science, University of Minnesota Duluth, Duluth, MN 55812, USA; \\ soufi001@d.umn.edu (M.S.); dowli026@d.umn.edu (D.D.) \\ * Correspondence: akhan@d.umn.edu
}

Received: 18 September 2020; Accepted: 21 October 2020; Published: 23 October 2020

\begin{abstract}
The electroencephalography (EEG) sensor has become a prominent sensor in the study of brain activity. Its applications extend from research studies to medical applications. This review paper explores various types of EEG sensors and their applications. This paper is for an audience that comprises engineers, scientists and clinicians who are interested in learning more about the EEG sensors, the various types, their applications and which EEG sensor would suit a specific task. The paper also lists the details of each of the sensors currently available in the market, their technical specs, battery life, and where they have been used and what their limitations are.
\end{abstract}

Keywords: EEG; EEG headset; EEG Cap

\section{Introduction}

An electroencephalography (EEG) sensor is an electronic device that can measure electrical signals of the brain. EEG sensors typically measure the varying electrical signals created by the activity of large groups of neurons near the surface of the brain over a period of time. They work by measuring the small fluctuations in electrical current between the skin and the sensor electrode, amplifying the electrical current, and performing any filtering, such as bandpass filtering [1].

Innovations in the field of medicine began in the early 1900s, prior to which there was little innovation due to the uncollaborative nature of the field of medicine. Innovation in diagnosis and treatment came from interdisciplinary advances in the applied sciences, such as those of physics and chemistry. One such innovation was the discovery of the small electrical currents produced by the brain and other organs. Measurement of electrical activity, such as in EEG, was not performed until after 1903, when the technique to measure the electrical activity of the heart was discovered by Willem Einthoven. This measurement technique was extended to the brain to extract the EEG signal [2].

\section{EEG Device Design Technology}

\subsection{Connection Types}

\subsubsection{Wired and Wireless Communications}

Wired and wireless EEG headsets transfer the data to a computer via a cable, wireless or Bluetooth connection, respectively. Wired EEG connections are more stable and often can transfer more data in a given time, but do not offer the freedom of movement provided by wireless connections. One of the main drawbacks of wireless EEG headsets is that, during the capture of brain data, the headset may lose its wireless connectivity and not record the data. Regardless of the connection type, the movement of cables and electrodes can cause artifacts in the EEG signal, as it can disrupt the connections between the electrodes and the scalp. 


\subsubsection{Electrode Connection}

EEG devices require a consistent electrical connection between the individual electrodes and the scalp of the individual wearing the device. This can be achieved in a variety of ways, some of which are listed below.

\subsubsection{Wet EEG Devices}

There are different types of wet EEG devices discussed below (Figure 1).

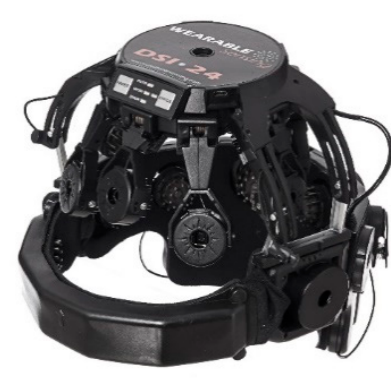

(a)

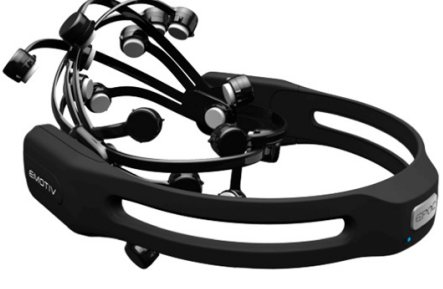

(b)

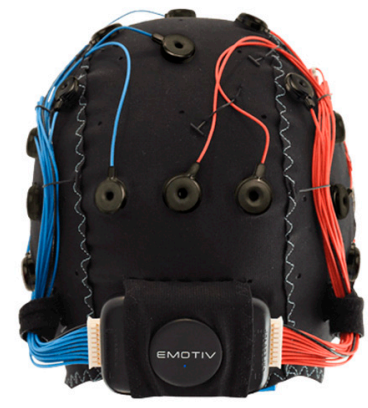

(c)

Figure 1. (a) dry, (b) saline solution, (c) gel-based.

Soft gel-based: Using this connection, electrodes connect with the scalp by applying conductive gel into the pocket of each electrode. After completion of an experiment, it is necessary to clean the headset by removing the gel and cleaning the electrodes. This is often done with alcohol because of its evaporative properties $[3,4]$.

Saline solution: Some of the EEG headsets require a conductive gel to help make low-impedance electrical contact between the skin and the sensor electrode. EEG headsets that have this technology connect electrodes by applying saline to each electrode [3,4].

Dry: Dry EEG devices do not use any gel or saline to connect the electrodes with the scalp, which makes it easier to record EEG data without the help of a trained technician [3,4]. Furthermore, its setup time is considerably shorter than wet headsets.

Others: Some EEG sensor connections types do not fit cleanly into either of these two categories. Conductive solid gel materials, such as those produced by Enobio, have also been used successfully in EEG devices.

\subsection{Differences between Dry and Wet Devices}

In January of 2019, researchers at the University of California, The Otto von Guericke University of Magdeburg, and The Hebrew University of Jerusalem performed a comparative analysis of the signal quality of dried wireless and wet wire EEG devices, and concluded that the quality of wireless dry devices is significantly comparable with the wired wet. Although some researchers observed that, for those activities that demand body movement like running/walking, wired wet sensors showed better performance [5,6]. This seems to indicate that wet sensors may be more resistant to movement artifacts, although more research needs to be conducted to fully understand which technology can provide more reliable data.

\subsection{Electrode Placement Standards}

The American clinical neurophysiology society suggested two international placements of electrodes on the scalp: 10-20 and 10-10 standards [7]. The numbers refer to the distances between adjacent electrodes placed on the skull. For example, for the 10-20 standard, the relative distance between an electrode and the underlying area on the skull is either $10 \%$ or $20 \%[7,8]$. The electrode 
location starts with a letter, followed by odd or even numbers to indicate the placement and the left or right side of the brain, including: $\mathrm{F}$ (frontal), $\mathrm{C}$ (central), $\mathrm{T}$ (temporal), $\mathrm{P}$ (posterior), and $\mathrm{O}$ (occipital) [9]. Figure 2 shows the name and position of each electrode in the 10-20 (black circles) and in 10-10 system (gray circles) [8]. The 10-20 system is suitable for both clinical and non-clinical studies and event-related potentials studies (ERPs) [8]. The 10-10 system is suitable for obtaining more detailed EEG data $[7,9]$.

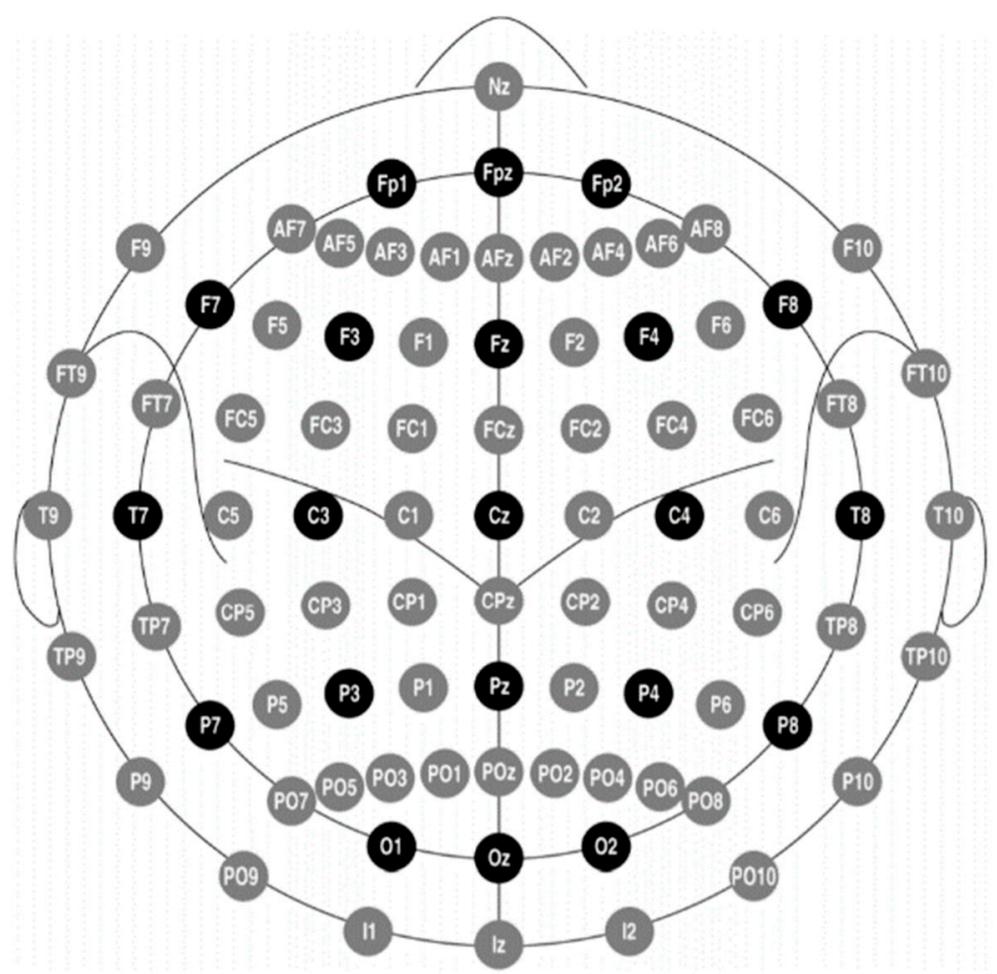

Figure 2. Electrode positions and labels in the $10 \pm 20$ system [8].

\subsection{EEG Devices with Several Sensors}

Some EEG devices are designed to capture both psychological (brain) and physiological (blood pressure, muscle activity, heart rate, etc.) data. These devices have one or more extra channels for capturing physiological signals such as Electrocardiogram (ECG), Electrooculography (EOG), Photoplethysmogram (PPG), and Electromyography (EMG).

ECG sensors record the heart's response during resting or physical activity.

EOG sensors measure human eye movements.

PPG sensors monitor blood volume changes.

EMG sensors collect muscle activity data.

Some EEG devices are equipped with motion sensors such as gyroscopes and accelerometers to capture head and body motion data. These can be used to measure, e.g., orientation, acceleration, and speed.

\section{Applications of EEG}

EEG devices can provide valuable information about human mental health states, thoughts, and imagination. Thus, researchers in different areas of research have utilized it. Figure 3 demonstrates five categories of EEG data applications and their relevant sub-categories. In a later section, EEG Headset Applications and Research Usages, a report of relative research use for various devices is provided as part of a table. 

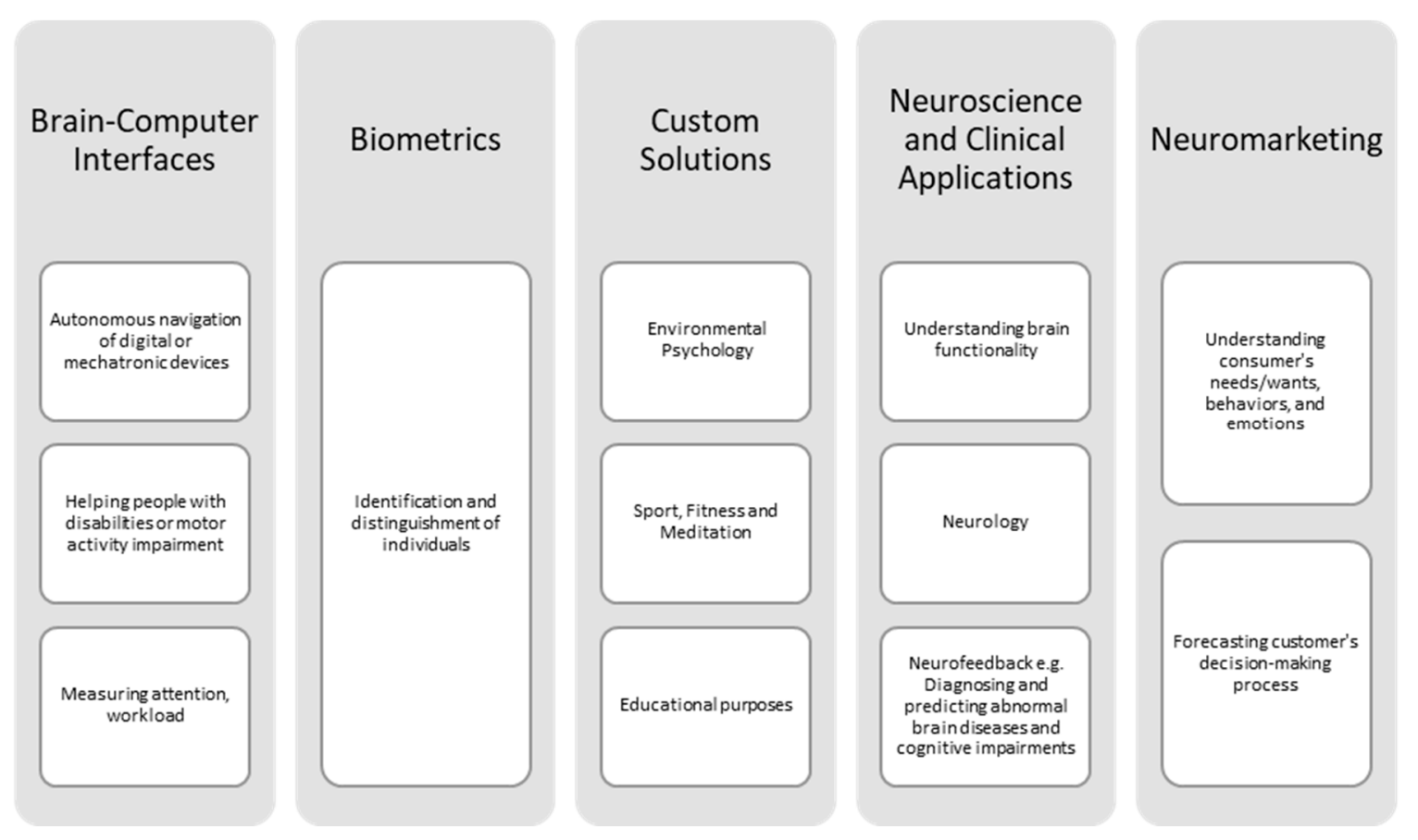

Figure 3. Electroencephalography (EEG) applications.

Brain-computer interfaces (BCI): BCIs, sometimes called brain-machine interfaces (BMIs), are one of the most common applications of EEG. BCIs use real-time EEG data to control and direct mechanical and electronic devices [10-17].

$\mathrm{BCI}$ devices are commonly used as a human-machine interface to help individuals with mild to severe motor disabilities, including those who are not able to communicate with others [10]. BCI devices designed for the disabled do not rely on muscle movements; instead, they use specific brain activity, imagining doing an activity, or concentrating on an object on-screen, and translate them into control functions and commands [10,11].

The most common BCI applications are listed below.

\section{Autonomous navigation of digital or mechatronic devices:}

- Real-time teleoperation of robotic body parts [18-22];

- Controlling and directing a robot [23-26], drone [27], dashboard of a vehicle [28], or a miniature or semi-automated car [29,30];

- Monitoring and controlling sensors inside of smart houses [31].

2. Helping people with disabilities or motor activity impairment:

- Control of mobile phone apps using eyewinks [32];

- Directing electrical wheelchair movement [33-36];

- Control of artificial body part such as prosthetic hand or arm [10,37,38];

- Recognizing a patient's attempt to move their body, e.g., stroke [39] and brain injury [40];

- Post-stroke motor rehabilitation using VR [41];

- Controlling a robot using body gestures [42];

- Mind-controlled dialing systems [43];

- Speech recognition system for people with speech disability [12,44];

- Mouse cursor control using imagined hand movement [45];

- Gaze controller for patients with neurodegenerative diseases [46]. 


\section{Neurogaming and Entertainment}

- Controlling a video game or virtual reality (VR) environment using body gesture and eye movement [47];

- Controlling fiber optic clothes or dresses [19].

Neurology: Real-time EEG signals can be used to provide immediate information about brain-wave activities. EEG data have been applied for diagnosing and predicting many abnormal brain diseases and cognitive impairments, listed below:

- Epilepsy [48];

- Parkinson's Disease [49];

- Memory problems like Alzheimer's [50];

- Language impairments such as Dyslexia [51];

- Attention Deficit Hyperactivity Disorder (ADHD) [52];

- Seizures [12];

- Schizophrenia [53];

- Autism in adults and children [54,55];

- Sleep disorders and insomnia [56,57];

- Anxiety [58];

- Post-traumatic stress disorder [10];

- Huntington's disease [59];

- Multiple sclerosis diagnosis [60];

- Amyotrophic lateral sclerosis [61];

- Traumatic brain injury (TBI) [62];

- Coma [63];

- Level of consciousness [64];

- Neurosurgery [65].

Neuroscience Research: Neuroscience attempts to understand the functionality of the nervous system. It allows clinical or non-clinical researchers to get an idea about how the brain acts when humans experience different emotional states and how the brain works in various mental states. Researchers have applied EEG devices in their studies in the below fields.

1. Cognitive neuroscience:

- $\quad$ Measuring cognitive load [28,66,67];

- Detecting differences between brain wave activity during suicidal and non-suicidal states [68]. Understanding brain activity during insight (insight is a moment where a human understands how to solve a puzzle or gains knowledge) [69];

- Analyzing brain workload during decision making or learning a new task $[60,70,71]$;

- $\quad$ Studying sleep pattern [72];

2. Behavioral neuroscience:

- $\quad$ Changing the workplace light and measuring brain alertness status [73];

- Measuring drowsiness or sleep detection for drivers and pilots [74];

- Measuring mental workload of deaf children exposed to a noisy environment during a word recognition task [75];

- Determining surgeon stress level while performing surgery [76];

- Identifying and reducing stress level [77];

- Environmental Psychology [78]. 
3. Neurophysiology:

- Measuring changes in brain after drinking alcohol [79];

- Detecting fatigue [74].

Neuroscience can also be applied to understanding human emotion in VR with/without the ability to touch the environment $[80,81]$ by displaying various types of media, such as:

- $\quad$ Real world or VR pictures [82];

- Images of nature and city environments [32];

- TV advertisements [36];

- Auditory stimuli [83];

- Multimedia [36,47,69], along with memory recall and dreams [84].

Neuromarketing or Consumer Neuroscience: Neuromarketing, one of the newest branches of the advertising industry, aims to understand the consumer's needs, behaviors and emotions, and forecast their decision-making processes [81,85-87]. Some neuromarketing research attempts to understand customers' preferences and expectations regarding a specific product [81] and their reaction to TV advertising by analyzing EEG signals [86,87].

Biometrics: Recognizing and distinguishing people using physiological or behavioral features such as fingerprint, voice, face, iris, gaze, gait and/or posture is called biometrics $[71,88,89]$. Studies show that EEG data can provide information about individuals' differences. Recently, cognitive and emotional brain status has been utilized for biometrics, meaning EEG data are used to identify people [89]. The main ideas behind why EEG-based biometric systems have received more attention recently relate to privacy compliance and robustness to spoofing attacks, as well as universality [88,89].

Custom Solutions and Neurofeedback (Neurotherapy): EEG devices have been applied in other areas of research to make a comfortable environment, improve well-being and life quality, and boost the learning process. Neurofeedback data can be used for either clinical or non-clinical research. Some customized EEG solutions are listed:

1. Sport, fitness and meditation: Monitoring health status and boosting quality of life using brain activity during exercise and listening to music [83].

\section{Educational purposes:}

i. Measuring the reading ability of students [84];

ii. Measuring confusion level during online lectures [90] or concentration level and cognitive workload when students are trying to solve a math puzzle [91] with the aim of designing intelligent tutor systems (ITS);

iii. Real-time brain visualization, which can have educational, training, or entertainment applications [92,93].

Figure 4 shows the most popular research topics of EEG data, as found by an internet search of "EEG" followed by each keyword in the pie chart of the figure. It is clear that after neuroscience, BCIs have received the most attention from researchers, and the percentage of studies on biometrics and neuro-marketing fields is relatively small. 


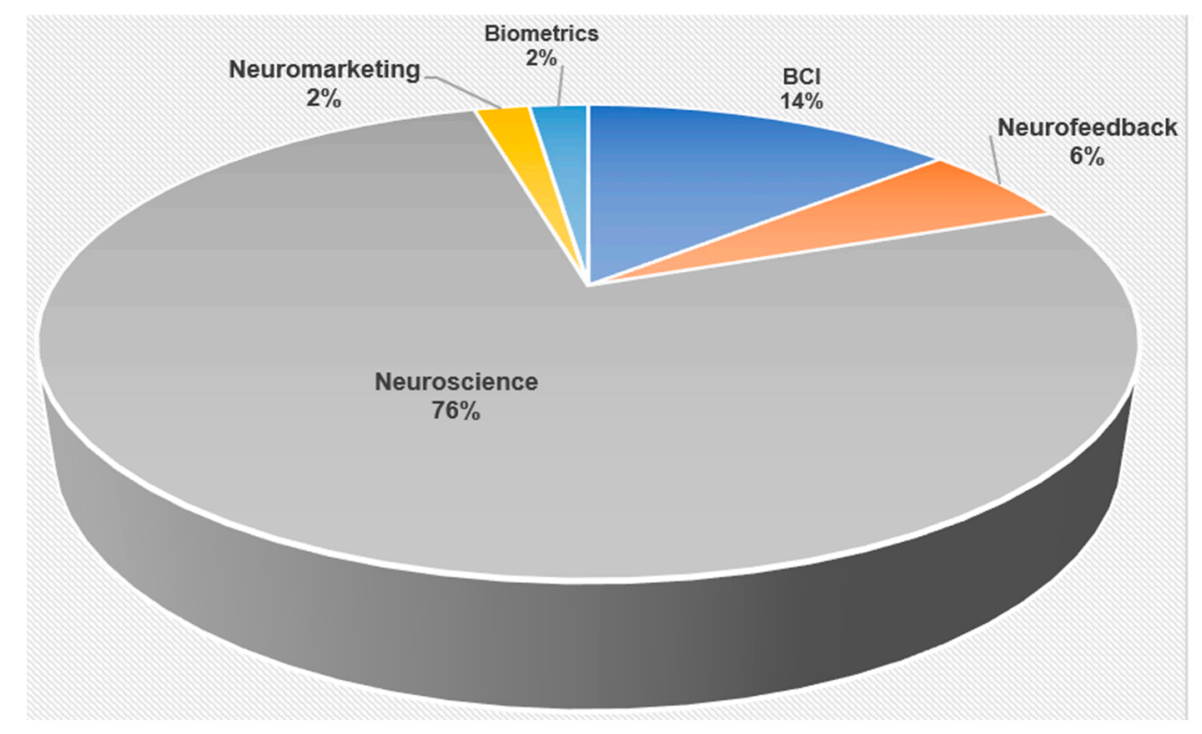

Figure 4. Percentage of each topic found through internet keyword search.

\section{EEG Headset Applications and Research Usages}

There are many commercially available EEG headsets. In the following, a set of tables shows the approximate applications for each headset, as well as their specifications and relative research use. In general, information was gathered from the company website for each product.

Table 1 indicates the applications that each EEG Headset has been used in, according to the producing company's reports.

Table 1. Recommended EEG application by manufactured company.

\begin{tabular}{|c|c|}
\hline Applications (Company Recommendation) & EEG Headset Product Name (Company) \\
\hline Neuroscience Research & $\begin{array}{ll}\text { - } & \text { DSI 24, DSI 7, DSI } 7 \text { Flex, VR300 (Wearable Sensing) } \\
\text { - } & \text { B-Alert X-Series (Advanced Brain Monitoring) } \\
\text { - } & \text { Enobio (Neuroelectrics) } \\
\text { - } & \text { Eego mylab (ANT Neuro) } \\
\text { - } & \text { Imec product } \\
\text { - } & \text { SMARTFONES, SMARTING (mBrainTrain) }\end{array}$ \\
\hline Neuromarketing & $\begin{array}{ll}\text { - } & \text { DSI 24, DSI } 7 \text { (Wearable Sensing) } \\
\text { - } & \text { B-Alert X-Series (Advanced Brain Monitoring) } \\
\text { - } & \text { SMARTFONES, SMARTING (mBrainTrain) }\end{array}$ \\
\hline Brain computer interface and Neurogaming & $\begin{array}{ll}\text { - } & \begin{array}{l}\text { DSI 24, DSI 7, VR300, NeuroCube, NeusenW } \\
\text { (Wearable Sensing) }\end{array} \\
\text { - } & \text { Epoc } \times, \text { Epoc }{ }^{+}, \text {Epoc Flex, INSIGHT (Emotiv) } \\
\text { - } & \text { B-Alert X-Series (Advanced Brain Monitoring) } \\
\text { - } & \text { Enobio (Neuroelectrics) } \\
\text { - } & \text { MindWave, MindWave Mobile } 2 \text { (NeuroSky) } \\
\text { - } & \text { BrainWaveBank products } \\
\text { - } & \text { NeusenW (wearable sensing) } \\
\text { - } & \text { Eego mylab, Eego }{ }^{\mathrm{TM}} \text { mini-series (ANT Neuro) } \\
\text { - } & \text { SMARTFONES, SMARTING (mBrainTrain) }\end{array}$ \\
\hline
\end{tabular}


Table 1. Cont.

\begin{tabular}{|c|c|}
\hline Applications (Company Recommendation) & EEG Headset Product Name (Company) \\
\hline Ergonomics and Biometrics & $\begin{array}{ll}\text { - } & \text { DSI 24, DSI } 7 \text { (Wearable Sensing) } \\
\text { - } & \text { B-Alert X-Series (Advanced Brain Monitoring) }\end{array}$ \\
\hline Neurofeedback & $\begin{array}{ll}\text { - } & \text { DSI 24, DSI } 7 \text { (Wearable Sensing) } \\
\text { Sleep studies: EEG Electrode Cap Kit (OPENBCI), } \\
\text { Sleep Profiler PSG2 }{ }^{\mathrm{TM}} \text {, Sleep Profiler }{ }^{\mathrm{TM}} \text { (Advanced } \\
\text { Brain Monitoring), Muse 2, Muse S (Muse }{ }^{\mathrm{TM}} \text { ), } \\
\text { LiveAmp (Brain Products), BE Micro (EB Neuro) } \\
\text { - } \quad \text { Enobio (Neuroelectrics) } \\
\text { - } \quad \text { MindWave Mobile 2 (NeuroSky) } \\
\text { - } \quad \text { Eego mylab (ANT Neuro) } \\
\text { - } \quad \text { SMec product } \\
\text { SMARTFNES, SMARTING (mBrainTrain) }\end{array}$ \\
\hline Biofeedback & $\begin{array}{l}\text { - Meditation and Sleep studies: Muse 2, Muse S (Muse } \\
\text { TM) } \\
\text { - Cardiology and Gastroenterology: BE Micro } \\
\text { (EB Neuro) }\end{array}$ \\
\hline Custom Solutions (e.g., Sports, education) & $\begin{array}{ll}\text { - } & \text { DSI } 7 \text { Flex (Wearable Sensing) } \\
\text { - } & \text { Enobio (Neuroelectrics) } \\
\text { - } & \text { MindWave, MindWave Mobile } 2 \text { (NeuroSky) } \\
\text { - } & \text { Eego mylab (ANT Neuro) }\end{array}$ \\
\hline
\end{tabular}

Table 2 provides information about the characteristics of the available EEG headsets $\backslash$ caps, such as number of channels, sampling rate, electrode connection type, headset preparation time (the amount of time it takes to prepare the headset/cap), and price. Some of the EEG devices are equipped with extra sensors, enabling them to track muscle activities (EMG), heart rate (ECG), eye movement (EOG), and blood pressure (PPG); these are indicated in the "Extra Sensors" column. The "Motion Sensors" column shows any EEG devices that have motion sensors, such as an accelerometer or gyroscope, for tracking body or head movement. The "Communication Mode" column indicates the way in which the sensor transfers its recorded data. If the EEG device is a wireless Bluetooth device, it works in a specific range, and outside of that distance, may not work correctly; this information is shown in the "Bluetooth range" column. The "Battery life" column shows how many hours the device is capable of working for using its wireless technology. For some companies, such as Compumedics Neuroscan, the listed EEG devices are only components of the full device, which is necessary to perform Electroencephalography, such as cap and amplifier, thus cannot be used without the purchase of separate hardware.

Some of the EEG devices have included open source software, allowing users to capture and analyze the data for free, while for some headset/caps, users need to pay subscription fees and/or purchase the related software; this information is denoted in the "Included Software" column. 
Table 2. Sensors information

\begin{tabular}{|c|c|c|c|c|c|c|c|}
\hline Company & Product Name & $\begin{array}{c}\text { Extra Sensors } \\
\text { (Optional) }\end{array}$ & Motion Sensors & $\begin{array}{c}\text { Communication } \\
\text { Mode(s) }\end{array}$ & $\begin{array}{l}\text { Bluetooth } \\
\text { Range }\end{array}$ & Included Software & $\begin{array}{c}\text { Battery Life (When } \\
\text { Applicable) }\end{array}$ \\
\hline \multirow{12}{*}{$\begin{array}{l}\text { Compumedics } \\
\text { Neuroscan }\end{array}$} & \multirow{3}{*}{ Quick-Cap Neo Net } & EOG & \multirow{3}{*}{ - } & \multirow{3}{*}{ - } & \multirow{3}{*}{ - } & \multirow{3}{*}{ - } & \multirow{3}{*}{ - } \\
\hline & & ECG & & & & & \\
\hline & & EMG & & & & & \\
\hline & \multirow{3}{*}{$\begin{array}{c}\text { Quick-cap Silicone } \\
\text { Array }\end{array}$} & EOG & \multirow{3}{*}{ - } & \multirow{3}{*}{ - } & \multirow{3}{*}{ - } & \multirow{3}{*}{ - } & \multirow{3}{*}{-} \\
\hline & & ECG & & & & & \\
\hline & & EMG & & & & & \\
\hline & \multirow{3}{*}{ Quick-Cap Hydro Net } & EOG & & \multirow{3}{*}{ - } & \multirow{3}{*}{-} & \multirow{3}{*}{-} & \multirow{3}{*}{-} \\
\hline & & ECG & - & & & & \\
\hline & & EMG & & & & & \\
\hline & \multirow{3}{*}{ Quick-Cap } & EOG & & \multirow{3}{*}{ - } & \multirow{3}{*}{-} & \multirow{3}{*}{ - } & \multirow{3}{*}{ - } \\
\hline & & ECG & - & & & & \\
\hline & & EMG & & & & & \\
\hline \multirow{11}{*}{ Wearable sensing } & \multirow[b]{2}{*}{ DSI 24} & EMG & & \multirow[b]{2}{*}{ Bluetooth Wireless } & \multirow[b]{2}{*}{$10 \mathrm{~m} / 30$ feet } & & \multirow[b]{2}{*}{ - } \\
\hline & & $\begin{array}{l}\text { EOG } \\
\text { ECG }\end{array}$ & $\begin{array}{l}\text { Accelerometer } \\
\quad(\mathrm{Opt})\end{array}$ & & & $\begin{array}{l}\text { Software and API } \\
\text { SOIton }\end{array}$ & \\
\hline & \multirow[b]{2}{*}{ DSI 7} & \multirow[b]{2}{*}{$\mathrm{N} / \mathrm{A}$} & Accelerometer & & & DSI-Streamer Data Acquisition & \\
\hline & & & $(\mathrm{Opt})$ & Bluetooth Wireless & $10 \mathrm{~m} / 30$ feet & Software and API & - \\
\hline & DSI 7 Flex & $\mathrm{N} / \mathrm{A}$ & Accelerometer & Bluetooth Wireless & $10 \mathrm{~m} / 30$ feet & DSI-Streamer Data Acquisition & - \\
\hline & & $\mathrm{N} / \mathrm{A}$ & (Opt) & & & Software and API & - \\
\hline & & & & & & DSI-Streamer Data Acquisition & \\
\hline & VR300 & N/A & $\begin{array}{l}\text { Accelerometer } \\
(\mathrm{Opt})\end{array}$ & Bluetooth Wireless & $10 \mathrm{~m} / 30$ feet & $\begin{array}{c}\text { Software, API, and Unity and } \\
\text { Unreal SDK for VR }\end{array}$ & - \\
\hline & & $N / A$ & 9-axis motion & Bluptooth Wiroloce & & & Un to $2 \mathrm{~b}$ \\
\hline & NeusenW & N/A & sensors & Bluetooth Wireless & - & - & Up to $2 \mathrm{~h}$ \\
\hline & NeuroCube & N/A & $\begin{array}{l}\text { 9-axis motion } \\
\text { sensors }\end{array}$ & Bluetooth Wireless & - & - & $\mathrm{Up}$ to $2 \mathrm{~h}$ \\
\hline & EPOC $X$ & $\mathrm{~N} / \mathrm{A}$ & 9-axis motion & Bluetooth Wireless & _ & EmotivPRO & Up to $9 \mathrm{~h}$ \\
\hline & & & sensors & Diuetootn vireless & - & Emotiv BrainViz & up to yn \\
\hline Emotiv & & & & & & EmotivPRO & \\
\hline & $\mathrm{EPOC}^{+}$ & N/A & $\begin{array}{c}\text { 3-axis } \\
\text { Accelerometer }\end{array}$ & Bluetooth Wireless & - & EmotivBCI & Up to $12 \mathrm{~h}$ \\
\hline & & & & & & Emotiv BrainViz & \\
\hline & MN8 & N/A & Motion sensors & Bluetooth Wireless & - & - & Up to $6 \mathrm{~h}$ \\
\hline
\end{tabular}


Table 2. Cont.

\begin{tabular}{|c|c|c|c|c|c|c|c|}
\hline Company & Product Name & $\begin{array}{l}\text { Extra Sensors } \\
\text { (Optional) }\end{array}$ & Motion Sensors & $\begin{array}{l}\text { Communication } \\
\text { Mode(s) }\end{array}$ & $\begin{array}{l}\text { Bluetooth } \\
\text { Range }\end{array}$ & Included Software & $\begin{array}{c}\text { Battery Life (When } \\
\text { Applicable) }\end{array}$ \\
\hline & INSIGHT & N/A & $\begin{array}{l}\text { 9-axis Motion } \\
\text { sensors }\end{array}$ & Bluetooth Wireless & - & - & Up to $9 \mathrm{~h}$ \\
\hline & EPOC Flex & N/A & $\begin{array}{c}\text { 3-axis } \\
\text { Accelerometer, } \\
\text { Magnetometer }\end{array}$ & $\begin{array}{l}\text { Bluetooth } \\
\text { Wireless }\end{array}$ & - & EmotivPRO & Up to $9 \mathrm{~h}$ \\
\hline \multirow{3}{*}{ OPEN BCI } & EEG Electrode Cap Kit & N/A & $\mathrm{N} / \mathrm{A}$ & Bluetooth Wireless & - & $\begin{array}{l}\text { OpenBCI's FREE open-source } \\
\text { software }\end{array}$ & - \\
\hline & $\begin{array}{l}\text { Ultracortex "Mark IV" } \\
\text { EEG headset }\end{array}$ & $\begin{array}{l}\text { EMG } \\
\text { ECG }\end{array}$ & $\begin{array}{c}\text { 3-axis } \\
\text { Accelerometer }\end{array}$ & Bluetooth Wireless & - & - & - \\
\hline & $\begin{array}{l}\text { OpenBCI Classroom } \\
\text { Bundle (5 kits) }\end{array}$ & $\begin{array}{l}\text { EMG } \\
\text { ECG }\end{array}$ & N/A & Bluetooth Wireless & - & - & - \\
\hline \multirow[t]{2}{*}{ Biosemi } & ActiveTwo & $\begin{array}{l}\text { EMG } \\
\text { ECG }\end{array}$ & N/A & Wired & - & LabVIEW & $\mathrm{N} / \mathrm{A}$ \\
\hline & ActiveOne & $\begin{array}{l}\text { EMG } \\
\text { ECG }\end{array}$ & N/A & Wired & - & & N/A \\
\hline \multirow{4}{*}{$\begin{array}{l}\text { Advanced Brain } \\
\text { Monitoring }\end{array}$} & Sleep Profiler ${ }^{\mathrm{TM}}$ & $\begin{array}{l}\text { EOG } \\
\text { EMG } \\
\text { ECG }\end{array}$ & N/A & Wireless & - & - & Up to $30 \mathrm{~h}$ \\
\hline & Sleep Profiler PSG2TM & $\begin{array}{l}\text { EEG } \\
\text { EOG } \\
\text { EMG }\end{array}$ & N/A & Wireless & - & - & Up to $30 \mathrm{~h}$ \\
\hline & Stat X-Series & $\begin{array}{l}\text { ECG } \\
\text { EOG } \\
\text { EMG }\end{array}$ & Accelerometer & Bluetooth Wireless & $10 \mathrm{~m}$ & $\begin{array}{l}\text { B-AlertLive } \\
\text { LabX }\end{array}$ & Up to $8 \mathrm{~h}$ \\
\hline & B-Alert X-Series & $\begin{array}{l}\text { ECG } \\
\text { EOG } \\
\text { EMG }\end{array}$ & Accelerometer & Bluetooth Wireless & $10 \mathrm{~m}$ & $\begin{array}{l}\text { B-AlertLive } \\
\text { LabX }\end{array}$ & Up to $8 \mathrm{~h}$ \\
\hline \multirow{3}{*}{ InteraXon } & Muse S & PPG & $\begin{array}{l}\text { Accelerometer } \\
\text { Gyroscope }\end{array}$ & Bluetooth Wireless & - & Muse App & $10 \mathrm{~h}$ \\
\hline & Muse S Bundle & PPG & $\begin{array}{l}\text { Accelerometer } \\
\text { Gyroscope }\end{array}$ & Bluetooth Wireless & - & Muse App & $10 \mathrm{~h}$ \\
\hline & Muse 2 & PPG & $\begin{array}{l}\text { Accelerometer } \\
\text { Gyroscope }\end{array}$ & Bluetooth Wireless & - & Muse App & $5 \mathrm{~h}$ \\
\hline
\end{tabular}


Table 2. Cont.

\begin{tabular}{|c|c|c|c|c|c|c|c|}
\hline Company & Product Name & $\begin{array}{l}\text { Extra Sensors } \\
\text { (Optional) }\end{array}$ & Motion Sensors & $\begin{array}{c}\text { Communication } \\
\text { Mode(s) }\end{array}$ & $\begin{array}{l}\text { Bluetooth } \\
\text { Range }\end{array}$ & Included Software & $\begin{array}{c}\text { Battery Life (When } \\
\text { Applicable) }\end{array}$ \\
\hline Neuroelectrics & Enobio & N/A & $\begin{array}{c}\text { 3-axis } \\
\text { Accelerometer }\end{array}$ & Bluetooth Wireless & - & $\begin{array}{c}\text { Enobio API } \\
\text { Matlab (EEGLAB Plugin) } \\
\text { Python (Neyp library) }\end{array}$ & $\mathrm{Up}$ to $20 \mathrm{~h}$ \\
\hline NeuroSky & MindWave Mobile 2 & ECG & $\mathrm{N} / \mathrm{A}$ & Bluetooth Wireless & $10 \mathrm{~m}$ & MindWave Mobile apps & $8 \mathrm{~h}$ \\
\hline Wearable Sensing & NeusenW & EOG & $\begin{array}{l}\text { 9-axis motion } \\
\text { sensor }\end{array}$ & Bluetooth Wireless & - & - & - \\
\hline \multirow{3}{*}{ ANT Neuro } & Eego $^{\mathrm{TM}}$ mylab & $\mathrm{N} / \mathrm{A}$ & $\mathrm{N} / \mathrm{A}$ & Bluetooth Wireless & - & API & Up to $5 \mathrm{~h}$ \\
\hline & Eego $^{\mathrm{TM}}$ sports & EMG & N/A & Bluetooth Wireless & - & API & $\mathrm{Up}$ to $5 \mathrm{~h}$ \\
\hline & Eego $^{\mathrm{TM}}$ mini-series & EMG & N/A & Bluetooth Wireless & - & API & $\mathrm{Up}$ to $5 \mathrm{~h}$ \\
\hline \multirow{4}{*}{ G.tec } & NAUTILUS FNIRS & N/A & $\begin{array}{c}\text { 3-axis } \\
\text { accelerometer }\end{array}$ & Bluetooth Wireless & $10 \mathrm{~m}$ & BSANALYZE & Up to $10 \mathrm{~h}$ \\
\hline & Nautilus Research & N/A & $\begin{array}{c}\text { 3-axis } \\
\text { accelerometer }\end{array}$ & Bluetooth Wireless & $0 \mathrm{~m}$ & BSANALYZE & $\mathrm{Up}$ to $6 \mathrm{~h}$ \\
\hline & Nautilus PRO & $\mathrm{N} / \mathrm{A}$ & $\begin{array}{c}\text { 3-axis } \\
\text { accelerometer }\end{array}$ & Bluetooth Wireless & $10 \mathrm{~m}$ & BSANALYZE & $\mathrm{Up}$ to $10 \mathrm{~h}$ \\
\hline & $\begin{array}{l}\text { G. nautilus multi } \\
\text { purpose }\end{array}$ & N/A & $\begin{array}{c}\text { 3-axis } \\
\text { accelerometer }\end{array}$ & Bluetooth Wireless & $10 \mathrm{~m}$ & BSANALYZE & - \\
\hline imec & - & N/A & - & Bluetooth Wireless & - & Qt-based, MS \& Android & Up to $8 \mathrm{~h}$ \\
\hline EB Neuro & BE Micro & - & N/A & Bluetooth Wireless & - & - & Up tp $72 \mathrm{~h}$ \\
\hline \multirow{3}{*}{ mBrain Train } & SMARTING & N/A & 3 axis gyroscope & Bluetooth Wireless & $10 \mathrm{~m}$ & API & Up to $5 \mathrm{~h}$ \\
\hline & SMARTFONES & $\mathrm{N} / \mathrm{A}$ & N/A & Bluetooth Wireless & - & API & - \\
\hline & SMARTING sleep & $\begin{array}{l}\text { ECG } \\
\text { EMG } \\
\text { EOG }\end{array}$ & $\begin{array}{c}9 \text { axis motion } \\
\text { sensor }\end{array}$ & Bluetooth Wireless & $10 \mathrm{~m}$ & API & Up to $15 \mathrm{~h}$ \\
\hline
\end{tabular}


Table 2. Cont.

\begin{tabular}{|c|c|c|c|c|c|c|c|}
\hline Company & Product Name & $\begin{array}{c}\text { Extra Sensors } \\
\text { (Optional) }\end{array}$ & Motion Sensors & $\begin{array}{c}\text { Communication } \\
\text { Mode(s) }\end{array}$ & $\begin{array}{c}\text { Bluetooth } \\
\text { Range }\end{array}$ & Included Software & $\begin{array}{c}\text { Battery Life (When } \\
\text { Applicable) }\end{array}$ \\
\hline \multirow{10}{*}{ Cognionics (CGX) } & & EOG & & & & & \\
\hline & & ECG & & & & & \\
\hline & Quick & EMG & N/A & Bluetooth Wireless & - & - & - \\
\hline & & PPG & & & & & \\
\hline & & GSR & & & & & \\
\hline & & EOG & & & & & \\
\hline & & ECG & & & & & \\
\hline & Mobile & EMG & N/A & Bluetooth Wireless & - & - & - \\
\hline & & PPG & & & & & \\
\hline & & GSR & & & & & \\
\hline \multirow{2}{*}{ Brain Product } & actiCAP (Slim \& Snap) & $\mathrm{N} / \mathrm{A}$ & $\mathrm{N} / \mathrm{A}$ & - & - & - & - \\
\hline & LiveAMP & N/A & $\mathrm{N} / \mathrm{A}$ & Bluetooth Wireless & - & - & - \\
\hline
\end{tabular}


In Table 3, publication numbers were found through an internet query of the name of the company followed by the product name; the numbers provided in the "Company name" column are the search results with only the company name. The relationship between the 'company-only' search results alongside the 'company-and-product' search results is important to note, as the nature of some company's names are such that the search results do not accurately reflect their influence on the research. To avoid searches that include individual words not reflective of the company or product being searched for, quotation marks were added around the search term. Additionally, the "MD" column indicates if the mentioned EEG device can be used for clinical research, meaning it has been FDA approved. This is indicated either by an $X$, meaning that the device has not been officially approved, or by a $\boldsymbol{V}$, indicating that the device has been approved.

Information about sampling rate, number of channels, set up time, and price is also provided. An N/A indicates that the information does not apply, and a "-" symbol indicates that the searchers were unable to ascertain the information. 
Table 3. List of EEG headsets

\begin{tabular}{|c|c|c|c|c|c|c|c|c|c|}
\hline Company & $\begin{array}{l}\text { Publications } \\
\text { (Company) }\end{array}$ & EEG Headset/Caps & $\begin{array}{c}\text { No. } \\
\text { Publications }\end{array}$ & $\mathrm{MD}^{1}$ & Sample Rate & No. Channels & $\begin{array}{c}\text { Electrode } \\
\text { Connection Type }\end{array}$ & $\begin{array}{c}\text { Set up Time: } \\
\text { Minutes(m) } \\
\text { or Seconds (s) }\end{array}$ & Price \\
\hline \multirow{4}{*}{$\begin{array}{l}\text { Compumedics } \\
\text { Neuroscan }\end{array}$} & & Quick_Cap Neo Net & & $x$ & & Up to 256 & Gel & - & - \\
\hline & & $\begin{array}{c}\text { Quick_Cap Silicone } \\
\text { Array }\end{array}$ & & $x$ & & Up to 256 & Saline & - & - \\
\hline & & $\begin{array}{c}\text { Quick_Cap Hydro } \\
\text { Net }\end{array}$ & & $x$ & & Up to 256 & Saline & - & - \\
\hline & & Quick-Cap & & $x$ & & Up to 256 & Gel & - & - \\
\hline \multirow{5}{*}{ Emotiv } & \multirow{5}{*}{8150} & INSIGHT & 362 & $x$ & $128 \mathrm{~Hz}$ & 5 & Semi-dry polymer & $1-2 \mathrm{~m}$ & $\$ 299$ \\
\hline & & EPOC $X$ & 1 & $x$ & $128 \mathrm{~Hz}$ & 14 & Wet (Saline) & $3-5 \mathrm{~m}$ & $\$ 849$ \\
\hline & & EPOC+ & 4370 & $x$ & $128 \mathrm{~Hz}$ & 14 & Saline soaked felt & $3-5 \mathrm{~m}$ & $\$ 699$ \\
\hline & & EPOC FLEX KIT & 0 & $x$ & $128 \mathrm{~Hz}$ & 32 & Saline/Gel & $15-30 \mathrm{~m}$ & $\$ 1699$ \\
\hline & & MN8 & 0 & $x$ & - & $\begin{array}{c}2(+4 \\
\text { reference })\end{array}$ & Dry & $30 \mathrm{~s}$ & - \\
\hline \multirow{4}{*}{ OpenBCI } & \multirow{4}{*}{835} & \multirow{3}{*}{ Ulracortex Mark IV } & \multirow{3}{*}{26} & \multirow{3}{*}{$x$} & \multirow{4}{*}{$125 \mathrm{HZ}$ or $250 \mathrm{~Hz}$} & \multirow{3}{*}{8 or 16} & \multirow{3}{*}{ Dry } & \multirow{3}{*}{$\sim 30 \mathrm{~s}$} & $\begin{array}{l}\text { Print-It-Yourself } \\
\text { (\$299.99-399.99) }\end{array}$ \\
\hline & & & & & & & & & $\begin{array}{c}\text { Unassembled } \\
(\$ 499.99-599.99)\end{array}$ \\
\hline & & & & & & & & & $\begin{array}{l}\text { Pro-Assembled } \\
\text { (\$699.99-849.99) }\end{array}$ \\
\hline & & $\begin{array}{c}\text { EEG Electrode Cap } \\
\text { Kit }\end{array}$ & 1 & $x$ & & 21 & Gel & $\sim 30 \mathrm{~s}$ & $\$ 399.99$ \\
\hline \multirow[t]{2}{*}{ BIOSEMI } & \multirow[t]{2}{*}{10,300} & ActiveTwo & 2650 & $x$ & $2,4,8,16 \mathrm{kHz}$ & 280 & Gel & - & $\begin{array}{l}€ 14,840 \\
€ 72,440 \\
\end{array}$ \\
\hline & & ActiveOne & 15 & - & - & Up to 144 & Gel & - & \\
\hline \multirow{4}{*}{$\begin{array}{l}\text { Advanced Brain } \\
\text { Monitoring }\end{array}$} & \multirow{4}{*}{2030} & $\begin{array}{l}\text { B-Allert (X10 or } \\
\text { X24) }\end{array}$ & 37 & $\boldsymbol{v}$ & $256 \mathrm{~Hz}$ & 9 and 24 & Dry & - & $\$ 1000-\$ 25,000$ \\
\hline & & Sleep Profiler & 2 & $v$ & - & Up to 8 & Dry & - & - \\
\hline & & $\begin{array}{l}\text { Sleep Profiler } \\
\text { PSG2TM }\end{array}$ & 0 & $v$ & - & Up to 13 & Dry & - & - \\
\hline & & Stat X-Series & 0 & $v$ & - & Up to 20 & Dry & - & - \\
\hline
\end{tabular}


Table 3. Cont.

\begin{tabular}{|c|c|c|c|c|c|c|c|c|c|}
\hline Company & $\begin{array}{c}\text { Publications } \\
\text { (Company) }\end{array}$ & EEG Headset/Caps & $\begin{array}{c}\text { No. } \\
\text { Publications }\end{array}$ & $\mathrm{MD}^{1}$ & Sample Rate & No. Channels & $\begin{array}{c}\text { Electrode } \\
\text { Connection Type }\end{array}$ & $\begin{array}{c}\text { Set up Time: } \\
\text { Minutes(m) } \\
\text { or Seconds (s) }\end{array}$ & Price \\
\hline \multirow{3}{*}{ InteraXon } & \multirow{3}{*}{1140} & Muse 2 & \multirow{3}{*}{158} & $x$ & $220 \mathrm{~Hz}$ or $500 \mathrm{~Hz}$ & 4 & Dry & - & $\$ 224.99$ \\
\hline & & Muse S Bunddle & & $x$ & - & 4 & Dry & - & $\$ 444.98$ \\
\hline & & Muse S & & $x$ & - & 4 & Dry & - & $\$ 344.99$ \\
\hline Neuroelectrics & 1200 & Enobio & 59 & $\checkmark$ & 500 SPS & $8,20,32$ & Dry/Wet & - & - \\
\hline \multirow{4}{*}{ G.tec } & \multirow{4}{*}{4950} & Nautilus Research & \multirow{4}{*}{16} & $x$ & $250 \mathrm{~Hz}$ or $500 \mathrm{~Hz}$ & $8,16,32,64$ & Gel & - & $\$ 1000-\$ 25,000$ \\
\hline & & NAUTILUS FNIRS & & $x$ & $250 \mathrm{~Hz}$ or $500 \mathrm{~Hz}$ & $8,16,32,64$ & Wet & - & - \\
\hline & & Nautilus PRO & & $\checkmark$ & $500 \mathrm{~Hz}$ & 8,16, and 32 & Dry/Wet & - & - \\
\hline & & $\begin{array}{l}\text { Nautilus multi- } \\
\text { purpose }\end{array}$ & & $x$ & $250 \mathrm{~Hz}$ or $500 \mathrm{~Hz}$ & $8,16,32,64$ & Wet & - & - \\
\hline \multirow{2}{*}{ Cognionics (CGX) } & \multirow{2}{*}{497} & QUICK & 49 & $x$ & $500 \mathrm{~Hz}$ or $1000 \mathrm{~Hz}$ & $8,20,30$ & Dry & - & $\$ 1000-\$ 25,000$ \\
\hline & & Mobile & 21 & $x$ & $500 \mathrm{~Hz}$ or $1000 \mathrm{~Hz}$ & 64,128 & Gel & - & - \\
\hline \multirow{3}{*}{ ANT Neuro } & \multirow{3}{*}{1110} & eego mylab & 8 & $x$ & $16 \mathrm{kHZ}$ & $32-256$ & Dry/Gel & - & $\$ 1000-\$ 25,000$ \\
\hline & & Eego $^{\mathrm{TM}}$ sports & 10 & $x$ & - & - & - & - & - \\
\hline & & Eego $^{\mathrm{TM}}$ mini-series & - & $x$ & - & - & - & $20 \mathrm{~m}$ & - \\
\hline \multirow{2}{*}{ Brain Products } & \multirow{2}{*}{11,700} & LiveAmp & 31 & $x$ & $250-1000 \mathrm{~Hz}$ & $8-64$ & Dry/Gel & & $\$ 1000-\$ 25,000$ \\
\hline & & ActiCAP & 899 & $x$ & - & - & - & - & - \\
\hline \multirow{7}{*}{ Wearable Sensing } & \multirow{7}{*}{1220} & $\begin{array}{c}\text { Dry Sensor } \\
\text { Interface Series }\end{array}$ & 0 & $X$ & $300-600 \mathrm{~Hz}$ & $2-21$ & Dry & $\sim 5$ min. & $\$ 1000-\$ 25,000$ \\
\hline & & VR300 & - & $x$ & $300 \mathrm{~Hz}$ & 7 & Dry & $1-3 \mathrm{~min}$ & - \\
\hline & & NeusenW & - & - & $\mathrm{Up}$ to $16 \mathrm{kHz}$ & $8-64$ & Wet & - & - \\
\hline & & NeuroCub & - & - & $16 \mathrm{kHz}$ & 8 & Wet & - & - \\
\hline & & DSI 24 & 18 & - & $300 \mathrm{~Hz}$ & 21 & Dry active hybrid & $3-5 \mathrm{~min}$ & - \\
\hline & & DSI 7 Flex & - & - & $300-600 \mathrm{~Hz}$ & - & Dry & - & - \\
\hline & & DSI 7 & 5 & - & $300-600 \mathrm{~Hz}$ & $2-6$ & Dry & $1-3 \mathrm{~min}$ & - \\
\hline NeuroSky & 4910 & MindWave Mobile 2 & 1510 & - & $150 \mathrm{~Hz}$ & 2 & Dry & - & - \\
\hline
\end{tabular}


Table 3. Cont.

\begin{tabular}{|c|c|c|c|c|c|c|c|c|c|}
\hline Company & $\begin{array}{l}\text { Publications } \\
\text { (Company) }\end{array}$ & EEG Headset/Caps & $\begin{array}{c}\text { No. } \\
\text { Publications }\end{array}$ & MD $^{1}$ & Sample Rate & No. Channels & $\begin{array}{c}\text { Electrode } \\
\text { Connection Type }\end{array}$ & $\begin{array}{l}\text { Set up Time: } \\
\text { Minutes(m) } \\
\text { or Seconds (s) }\end{array}$ & Price \\
\hline BrainWave Bank & 7 & - & - & - & - & 16 & - & $\sim 5 \mathrm{~m}$ & - \\
\hline imec & $92,200^{2}(1690)$ & EEG Headset & 17 & - & $128,256,1028 \mathrm{~Hz}$ & 8 & Dry & - & - \\
\hline EBNeuro & 367 & BE Micro & 57 & - & - & - & - & - & - \\
\hline \multirow{3}{*}{ mBrainTrain } & \multirow{3}{*}{159} & SMARTING & 99 & - & $250-500 \mathrm{~Hz}$ & 24 & - & - & - \\
\hline & & SMARTFONES & 1 & - & Up to $1000 \mathrm{~Hz}$ & 11 & Semi-dry & - & - \\
\hline & & SMARTING sleep & - & - & $250-500 \mathrm{~Hz}$ & 17 & Dry & - & - \\
\hline
\end{tabular}

${ }^{1}$ MD certified might be for diagnostic or for Clinical Treatment. ${ }^{2}$ The results for the "imec" query were deemed too vague to be of use, so the term "EEG" was added outside of the quotation marks; the parenthetical number is the result of this search. 


\section{Discussion}

To select an EEG device, it is necessary to look at a variety of factors, some of which are listed below:

- Designing Technology Dry/Wet (saline or gel): Unfortunately, there are few studies done on this area. Recently, some researchers $[35,37]$ compared a dry and wet headset for their research and they concluded that although the selected dry EEG headset was more robust to line noise, it contained more artifacts;

- Setup time: Regardless of the connection type being used, the setup time for EEG electrodes tends to be longer than for most physiological sensors. Saline-based sensors are usually selected for their ease of use and quick setup time, relative to gel-based sensors. Gel based EEG devices demand a larger amount of time, relative to other connection methods, to apply, while the saline headset does not take much time to set up. Cleaning saline headsets after using them takes less time than gel-based sensors. The gel also sticks to the hair of participants, which could be uncomfortable and inconvenient for users;

- Signal quality and stability: Quality of the captured EEG data depends on several factors: connection stability, losing connection with the scalp, and wireless, which are described below:

1. Losing Connection with the Scalp: The quality of the recorded EEG data highly depends on the connection between electrodes and the scalp. Gel-based sensors are usually chosen for their stability of connection and longevity, as the wet or gel-based sensors maintain a more stable connection for several hours, while wet and dry EEG headsets may lose humidity during an experiment, which can lead to a decline in signal quality. To have stable, high-quality and reliable EEG data, it is necessary to make sure that all relevant electrodes are connected and do not lose their connection during experiments by reapplying the saline solution to the electrodes, as the solution evaporates over time. In order to maintain a stable connection over long periods, it is necessary to reapply the saline solution to the electrodes, as the solution evaporates over time;

2. Wireless Connectivity: Wireless EEG devices can pose a security risk to the data of the participant, as any movement of cables could potentially induce the data during transfer. Because of this, wireless EEG devices should necessarily require encryption of the data prior to wireless transfer.

- Headset Size: Most EEG devices are limited in their size adjustability, and may thus require multiple different caps or headsets in order to fit experiments and studies which collect data of individuals with large head-size discrepancies, increasing the overall price;

- Battery Life: Wireless EEG devices are most often battery-operated and, as such, are subject to potential loss of data if the current battery charge falls below threshold levels. Ensuring that batteries will be operational throughout long studies can be difficult, and the necessity of ensuring batteries are charged increases the complexity of data-gathering using EEG devices. Battery life has a negative correlation with the amount of sensory information they provide; as more information is given, the battery time decreases, which means that the research focused on long-term study of brain activities should try to rely on less sensory information, if possible;

- Sensitivity to external noise/artifacts: When collecting EEG data, it is important to ask the participants to sit in a relaxed manner because any movement of the body can cause artifacts in the data. To obtain high-quality data and better results, artifacts such as muscle and eye movement, eye blink, and line noise need to be pre-processed and artifacts should be omitted before doing any data analysis;

- Price: Most of the EEG devices designed for medical purposes like Neurofeedback and neuroscience are expensive; 
- API/Software Used by Device: The software which accompanies an EEG headset can be complicated for researchers without prior extensive knowledge about brain activity, as well as knowledge of filtering and analysis techniques. The software, which is utilized by an EEG device, can have adverse effects on the ease and reliability of experiments, as well as the overall cost. In addition to research on the quality of the EEG device itself, care should be taken to understand if the software it makes use of is within acceptable cost and quality levels. Open Source software tends to be more secure, but has less built-in support for newer users, whereas integrated proprietary software tends to have better support, but is more costly. Depending on the EEG software, users may be given access to raw EEG data that has not been modified, processed data that has been modified after recording by the software in some way, or to both raw and processed data;

- Comfort to user: Wireless dry or saline solution EEG devices are more convenient for the user because of their flexibility of movement, lower setup time, and no need for cleaning the user hairs after the experiment like in gel-based solution;

- CE/FDA approved: Most of the listed commercial EEG sensors have not been CE/FDA approved. A list of EEG headsets that can be utilized for clinical treatments is given in the "MD" column of Table 3.

\section{Conclusions and Future Research}

EEG devices are quickly becoming less expensive and more accessible to the open market, which should allow for more commercial and personal use of the data. Because of their widespread availability, many considerations should be made before a decision is made to purchase and use a device. Along with price, other factors that need to be considered are the battery life of an EEG device, available software for data analysis, and common uses of the device in research areas, especially where your own research may apply.

For future research, areas like biometrics and neuro-marketing currently have very little related research, and thus may be good avenues for further study.

Author Contributions: All authors have made a substantial contribution to the study and manuscript preparation. Author contribution are as follows: A.K. (revising the manuscript and final approval of manuscript), M.S. and D.D. (reviewing available papers, looking for the related EEG devices and potentials applications, and revising the manuscript). All authors have read and agreed to the published version of the manuscript.

Funding: This research received no external funding.

Conflicts of Interest: The authors declare no conflict of interest.

\section{References}

1. Sullivan, T.J.; Deiss, S.R.; Cauwenberghs, G. A low-noise, non-contact EEG/ECG sensor. In Proceedings of the 2007 IEEE Biomedical Circuits and Systems Conference, Montreal, QC, Canada, 27-30 November 2007; pp. 154-157.

2. Bronzino, J.D. Biomedical Engineering Handbook; CRC Press: Boca Raton, FL, USA, 1999; Volume 2.

3. Kam, J.W.Y.; Griffin, S.; Shen, A.; Patel, S.; Hinrichs, H.; Heinze, H.J.; Deouell, L.Y.; Knight, R.T. Systematic comparison between a wireless EEG system with dry electrodes and a wired EEG system with wet electrodes. Neuroimage 2019, 184, 119-129. [CrossRef] [PubMed]

4. Bastos, T.; Müller, S.; Godinez Tello, R.; Ferreira, A. Comparison between wire and wireless EEG acquisition systems based on SSVEP in an Independent-BCI. In Proceedings of the 2014 36th Annual International Conference of the IEEE Engineering in Medicine and Biology Society, EMBC 2014, Chicago, IL, USA, 26-30 August 2014. [CrossRef]

5. Hinrichs, H.; Scholz, M.; Baum, A.K.; Kam, J.W.; Knight, R.T.; Heinze, H.J. Comparison between a wireless dry electrode EEG system with a conventional wired wet electrode EEG system for clinical applications. Sci. Rep. 2020, 10, 5218. [CrossRef] [PubMed] 
6. Gu, X.; Cao, Z.; Jolfaei, A.; Xu, P.; Wu, D.; Jung, T.; Lin, C. EEG-based Brain-Computer Interfaces (BCIs): A Survey of Recent Studies on Signal Sensing Technologies and Computational Intelligence Approaches and their Applications. arXiv 2020, arXiv:2001.11337.

7. Acharya, J.N.; Hani, A.; Cheek, J.; Thirumala, P.; Tsuchida, T.N. American Clinical Neurophysiology Society Guideline 2: Guidelines for Standard Electrode Position Nomenclature. J. Clin. Neurophysiol. 2016, 33, 308-311. [CrossRef] [PubMed]

8. Oostenveld, R.; Praamstra, P. The five percent electrode system for high-resolution EEG and ERP measurements. Clin Neurophysiol. 2001, 112, 713-719. [CrossRef]

9. Abhang, P.A.; Gawali, B.W.; Mehrotra, S.C. Introduction to EEG- and Speech-Based Emotion Recognition, 1st ed.; Academic Press Inc.: Cambridge, MA, USA, 2016.

10. Schalk, G.; McFarland, D.J.; Hinterberger, T.; Birbaumer, N.; Wolpaw, J.R. BCI2000: A general-purpose brain-computer interface (BCI) system. IEEE Trans. Biomed. Eng. 2004, 51, 1034-1043. [CrossRef]

11. Guger, C.; Allison, B.; Edlinger, G. Brain-Computer Interface Research: A State-Of-The-Art Summary; Springer: Berlin, Germany; New York, NY, USA, 2013.

12. Guger, C.; Allison, B.; Leuthardt, E.C. Brain-Computer Interface Research: A State-Of-The-Art Summary 2; Springer: Heidelberg, Germany, 2014.

13. Guger, C.; Allison, B.; Leuthardt, E.C. Brain-Computer Interface Research: A State-Of-The-Art Summary 3; Springer: Cham, Switzerland, 2014.

14. Guger, C.; Muller-Putz, G.; Allison, B. Brain-Computer Interface Research: A State-Of-The-Art Summary 4; Springer: Cham, Switzerland, 2015.

15. Guger, C.; Allison, B.; Ushiba, J. Brain-Computer Interface Research: A State-Of-The-Art Summary 5; Springer: Cham, Switzerland, 2017. [CrossRef]

16. Guger, A.; Lebedev, G.; Christoph, A.; Lebedev, M. Brain-Computer Interface Research: A State-Of-The-Art Summary 6; Springer: Cham, Switzerland, 2017.

17. Guger, C.; Mrachacz-Kersting, N.; Allison, B.Z. Brain-Computer Interface Research: A State-Of-The-Art Summary 7; Springer: Cham, Switzerland, 2019.

18. Kline, A.; Desai, J. SIMULINK ${ }^{\circledR}$ based robotic hand control using Emotiv ${ }^{\mathrm{TM}}$ EEG headset. In Proceedings of the 2014 40th Annual Northeast Bioengineering Conference (NEBEC), Boston, MA, USA, 25-27 April 2014; pp. 1-2.

19. Ashford, R. ThinkerBelle EEG Amplifying Dress; Association for Computing Machinery: New York, NY, USA, 2015; pp. 607-612. [CrossRef]

20. Ranky, G.N.; Adamovich, S. Analysis of a commercial EEG device for the control of a robot arm. In Proceedings of the 2010 IEEE 36th Annual Northeast Bioengineering Conference (NEBEC), New York, NY, USA, 26-28 March 2010; pp. 1-2. [CrossRef]

21. Li, Y.; Zhou, G.; Graham, D.; Holtzhauer, A. Towards an EEG-based brain-computer interface for online robot control. Multimed. Tools Appl. 2016, 75, 7999-8017. [CrossRef]

22. Buck, T.; Matthews, A.; Alba-Flores, R. Robotic Arm Control through the Use of Human Machine Interfaces and Brain Signals; SoutheastCon: Huntsville, AL, USA, 2019; pp. 1-4. [CrossRef]

23. Chowdhury, P.; Shakim, S.S.K.; Karim, M.R.; Rhaman, M.K. Cognitive efficiency in robot control by Emotiv EPOC. In Proceedings of the 2014 International Conference on Informatics, Electronics \& Vision (ICIEV), Dhaka, Bangladesh, 23-24 May 2014; pp. 1-6.

24. Grude, S.; Freeland, M.; Yang, C.; Ma, H. Controlling mobile Spykee robot using Emotiv Neuro headset. In Proceedings of the 32nd Chinese Control Conference, Xi'an, China, 26-28 July 2013; pp. 5927-5932.

25. Perera, C.J.; Naotunna, I.; Sadaruwan, C.; Gopura, R.A.R.C.; Lalitharatne, T.D. SSVEP based BMI for a meal assistance robot. In Proceedings of the 2016 IEEE International Conference on Systems, Man, and Cybernetics (SMC), Budapest, Hungary, 9-12 October 2016.

26. Katona, J.; Ujbanyi, T.; Sziladi, G.; Kovari, A. Speed control of Festo Robotino mobile robot using NeuroSky MindWave EEG headset based brain-computer interface. In Proceedings of the 2016 7th IEEE International Conference on Cognitive Infocommunications (CogInfoCom), Wroclaw, Poland, 16-18 October 2016; pp. 000251-000256.

27. Pan, P.; Tan, G.; Phyo Wai, A.A. Evaluation of Consumer-Grade EEG Headsets for BCI Drone Control. Available online: http://oar.a-star.edu.sg:80/jspui/handle/123456789/2149 (accessed on 18 August 2020). 
28. Cernea, D.; Olech, P.-S.; Ebert, A.; Kerren, A. Controlling in-vehicle systems with a commercial EEG headset: Performance and cognitive load. Open Access Ser. Inform. 2012, 27, 113-122. [CrossRef]

29. Poorna, S.S.; Baba, P.S.; Ramya, G.L.; Poreddy, P.; Aashritha, L.S.; Nair, G.J.; Renjith, S. Classification of EEG based control using ANN and KNN-A comparison. In Proceedings of the 2016 IEEE International Conference on Computational Intelligence and Computing Research (ICCIC), Chennai, India, 15-17 December 2016; pp. 1-6. [CrossRef]

30. Göhring, D.; Latotzky, D.; Wang, M.; Rojas, R. Semi-autonomous Car Control Using Brain Computer Interfaces. In Intelligent Autonomous Systems 12. Advances in Intelligent Systems and Computing; Lee, S., Cho, H., Yoon, K.J., Lee, J., Eds.; Springer: Berlin/Heidelberg, Germany, 2013; Volume 194.

31. Shivappa, V.K.K.; Luu, B.; Solis, M.; George, K. Home automation system using brain computer interface paradigm based on auditory selection attention. In Proceedings of the 2018 IEEE International Instrumentation and Measurement Technology Conference (I2MTC), Houston, TX, USA, 14-17 May 2018; pp. 1-6.

32. Campbell, A.; Choudhury, T.; Hu, S.; Lu, H.; Mukerjee, M.; Rabbi, M.; Raizada, R. NeuroPhone: Brain-Mobile Phone Interface using a Wireless EEG Headset. In Proceedings of the Second ACM SIGCOMM Workshop on Networking, Systems and Applications on Mobile Handhelds, New Delhi, India, 30 August-3 September 2010; pp. 3-8. [CrossRef]

33. Mistry, K.S.; Pelayo, P.; Anil, D.G.; George, K. An SSVEP based brain computer interface system to control electric wheelchairs. In Proceedings of the 2018 IEEE International Instrumentation and Measurement Technology Conference (I2MTC), Houston, TX, USA, 14-17 May 2018; pp. 1-6.

34. Siswoyo, A.; Arief, Z.; Sulistijono, I.A. Application of Artificial Neural Networks in Modeling Direction Wheelchairs Using Neurosky Mindset Mobile (EEG) Device. EMITTER Int. J. Eng. Technol. 2017, 5, 170-191. [CrossRef]

35. Carrino, F.; Dumoulin, J.; Mugellini, E.; Khaled, O.A.; Ingold, R. A self-paced BCI system to control an electric wheelchair: Evaluation of a commercial, low-cost EEG device. In Proceedings of the 2012 ISSNIP Biosignals and Biorobotics Conference: Biosignals and Robotics for Better and Safer Living (BRC), Manaus, Brazil, 9 January 2012; pp. 1-6.

36. Mirza, I.A.; Tripathy, A.; Chopra, S.; D'Sa, M.; Rajagopalan, K.; D'Souza, A.; Sharma, N. Mind-controlled wheelchair using an EEG headset and arduino microcontroller. In Proceedings of the 2015 International Conference on Technologies for Sustainable Development (ICTSD), Mumbai, India, 4-6 February 2015; pp. 1-5.

37. Kasim, M.; Low, C.; Ayub, M.; Zakaria, C.; Ayuni, N.; Salleh, M.; Johar, K.; Hamli, H. User-Friendly LabVIEW GUI for Prosthetic Hand Control Using Emotiv EEG Headset. Procedia Comput. Sci. 2017, 105, 276-281. [CrossRef]

38. Beyrouthy, T.; al Kork, S.K.; Korbane, J.A.; Abdulmonem, A. EEG Mind controlled Smart Prosthetic Arm. In Proceedings of the 2016 IEEE International Conference on Emerging Technologies and Innovative Business Practices for the Transformation of Societies (EmergiTech), Balaclava, Mauritius, 1-6 August 2016; pp. 404-409.

39. Spicer, R.; Anglin, J.; Krum, D.M.; Liew, S. REINVENT: A low-cost, virtual reality brain-computer interface for severe stroke upper limb motor recovery. In Proceedings of the 2017 IEEE Virtual Reality (VR), Los Angeles, CA, USA, 18-22 March 2017; pp. 385-386.

40. Laibow, R.E.; Stubblebine, A.N.; Sandground, H.; Bounias, M. EEG-NeuroBioFeedback Treatment of Patients with Brain Injury: Part 2: Changes in EEG Parameters versus Rehabilitation. J. Neurother. 2002, 5, 45-71. [CrossRef]

41. Steinisch, M.; Tana, M.G.; Comani, S. A Post-Stroke Rehabilitation System Integrating Robotics, VR and High-Resolution EEG Imaging. IEEE Trans. Neural Syst. Rehabil. Eng. 2013, 21, 849-859. [CrossRef] [PubMed]

42. Jang, W.A.; Lee, S.M.; Lee, D.H. Development BCI for individuals with severely disability using EMOTIV EEG headset and robot. In Proceedings of the 2014 International Winter Workshop on Brain-Computer Interface (BCI), Jeongsun-kun, Korea, 17-19 February 2014; pp. 1-3. [CrossRef]

43. Jijun, T.; Peng, Z.; Ran, X.; Lei, D. The portable P300 dialing system based on tablet and Emotiv Epoc headset. In Proceedings of the 2015 37th Annual International Conference of the IEEE Engineering in Medicine and Biology Society (EMBC), Milan, Italy, 25-29 August 2015; pp. 566-569. [CrossRef]

44. Jacoby, J.D.; Tory, M.; Tanaka, J. Evoked response potential training on a consumer EEG headset. In Proceedings of the 2015 IEEE Pacific Rim Conference on Communications, Computers and Signal Processing (PACRIM), Victoria, BC, USA, 24-26 August 2015; pp. 485-490. 
45. Alomari, M.; Abubaker, A.; Turani, A.; Baniyounes, A.; Manasreh, A. EEG Mouse: A Machine Learning-Based Brain Computer Interface. Int. J. Adv. Comput. Sci. Appl. 2014, 5, 193-198. [CrossRef]

46. Aloise, F.; Aricò, P.; Schettini, F.; Salinari, S.; Mattia, D.; Cincotti, F. Asynchronous gaze-independent event-related potential-based brain-computer interface. Artif. Intell. Med. 2013, 59, 61-69. [CrossRef] [PubMed]

47. Hawsawi, O.; Semwal, S.K. EEG headset supporting mobility impaired gamers with game accessibility. In Proceedings of the 2014 IEEE International Conference on Systems, Man, and Cybernetics (SMC), San Diego, CA, USA, 5-8 October 2014; pp. 837-841. [CrossRef]

48. Acharya, U.R.; Subbhuraam, V.S.; Goutham, S.; Martis, R.; Suri, J. Automated EEG analysis of epilepsy: A review. Knowl. Based Syst. 2013, 45, 147-165. [CrossRef]

49. Klassen, B.T.; Hentz, J.G.; Shill, H.A.; Driver-Dunckley, E.; Evidente, V.G.; Sabbagh, M.N.; Adler, C.H.; Caviness, J.N. Quantitative EEG as a predictive biomarker for Parkinson disease dementia. Neurology 2011, 77, 118-124. [CrossRef]

50. Melissant, C.; Ypma, A.; Frietman, E.; Stam, C. A method for detection of Alzheimer's disease using ICA-enhanced EEG measurements. Artif. Intell. Med. 2005, 33, 209-222. [CrossRef]

51. Rippon, G.; Brunswick, N. Trait and State EEG Indices of Information Processing in Developmental Dyslexia. Int. J. Psychophysiol. 2000, 36, 251-265. [CrossRef]

52. Lansbergen, M.M.; van Dongen-Boomsma, M.; Buitelaar, J.K.; Slaats-Willemse, D. ADHD and EEG-neurofeedback: A double-blind randomized placebo-controlled feasibility study. J. Neural Transm. 2011, 118, 275-284. [CrossRef]

53. Itil, T.M. Qualitative and quantitative EEG findings in schizophrenia. Schizophr. Bull. 1977, 3, 61-79. [CrossRef]

54. Alturki, F.; Alsharabi, K.; Abdurraqeeb, A.; Aljalal, M. EEG Signal Analysis for Diagnosing Neurological Disorders Using Discrete Wavelet Transform and Intelligent Techniques. Sensors 2020, 20, 2505. [CrossRef] [PubMed]

55. Kang, J.; Han, X.; Song, J.; Niu, Z.; Li, X. The identification of children with autism spectrum disorder by SVM approach on EEG and eye-tracking data. Comput. Biol. Med. 2020, 120, 103722. [CrossRef] [PubMed]

56. Parrino, L.; Ferri, R.; Bruni, O.; Terzano, M. Cyclic alternating pattern (CAP): The marker of sleep instability. Sleep Med. Rev. 2011, 16, 27-45. [CrossRef] [PubMed]

57. Siddiqui, M.M.; Srivastava, G.; Saeed, S.H. Diagnosis of insomnia sleep disorder using short time frequency analysis of PSD approach applied on EEG signal using channel ROC-LOC. Sleep Sci. 2016, 9, 186-191. [CrossRef]

58. Wang, Y. EEG-based Anxious Personality Prediction. Ph.D. Thesis, University of Otago, Dunedin, New Zealand, 2020. Available online: http://hdl.handle.net/10523/10044 (accessed on 10 December 2019).

59. Painold, A.; Anderer, P.; Holl, A.K.; Letmaier, M.; Saletu-Zyhlarz, G.M.; Saletu, B.; Bonelli, R.M. Comparative EEG mapping studies in Huntington's disease patients and controls. J. Neural Transm. 2010, 117, 1307-1318. [CrossRef]

60. Raeisi, K.; Mohebbi, M.; Khazaei, M.; Seraji, M.; Younesi, A. Phase-synchrony evaluation of EEG signals for Multiple Sclerosis diagnosis based on bivariate empirical mode decomposition during a visual task. Comput. Biol. Med. 2019, 117, 103596. [CrossRef]

61. Maruyama, Y.; Yoshimura, N.; Rana, A.; Malekshahi, A.; Tonin, A.; Jaramillo-Gonzalez, A.; Birbaumer, N.; Chaudhary, U. Electroencephalography of completely locked-in state patients with amyotrophic lateral sclerosis. Neurosci Res. 2020. [CrossRef]

62. Ianof, J.N.; Anghinah, R. Traumatic brain injury: An EEG point of view. Dement. Neuropsychol. 2017, 11, 3-5. [CrossRef]

63. Young, G. Bryan The EEG in Coma. J. Clin. Neurophysiol. 2000, 17, 473-485. [CrossRef]

64. Simon, C.W.; Emmons, W.H. EEG, Consciousness, and Sleep. Science 1956, 124, 1066-1069. Available online: www.jstor.org/stable/1753085 (accessed on 6 June 2020). [CrossRef]

65. Cerutti, S.; Liberati, D.; Avanzini, G.; Silvana, F.; Panzica, F. Classification of the EEG during neurosurgery. Parametric identification and Kalman filtering compared. J. Biomed. Eng. 1986, 8, 244-254. [CrossRef] 
66. Das, R.; Chatterjee, D.; Das, D.; Sinharay, A.; Sinha, A. Cognitive load measurement-A methodology to compare low cost commercial EEG devices. In Proceedings of the 2014 International Conference on Advances in Computing, Communications and Informatics (ICACCI), New Delhi, India, 24-27 September 2014; pp. 1188-1194. [CrossRef]

67. Mills, C.; Fridman, I.; Soussou, W.; Waghray, D.; Olney, A.; D'Mello, S. Put Your Thinking Cap on: Detecting Cognitive Load Using EEG during Learning; Association for Computing Machinery: New York, NY, USA, 2017; pp. 80-89. [CrossRef]

68. Graae, F.; Tenke, C.; Bruder, G.; Rotheram, M.J.; Piacentini, J.; Castro-Blanco, D.; Leite, P.; Towey, J. Abnormality of EEG alpha asymmetry in female adolescent suicide attempters. Biol. Psychiatry 1996, 40, 706-713. [CrossRef]

69. Cernea, D.; Kerren, A.; Ebert, A. Detecting Insight and Emotion in Visualization Applications with a Commercial EEG Headset. Proceedings of SIGRAD 2011, Evaluations of Graphics and Visualization-Efficiency, Usefulness, Accessibility, Usability, Stockholm, Sweden, 17-18 November 2011.

70. Borghini, G.; Arico, P.; Astolfi, L.; Toppi, J.; Cincotti, F.; Mattia, D.; Cherubino, P.; Vecchiato, G.; Maglione, A.G.; Graziani, I.; et al. Frontal EEG theta changes assess the training improvements of novices in flight simulation tasks. Annu. Int. Conf. IEEE Eng. Med. Biol. Soc. 2013, 2013, 6619-6622. [CrossRef] [PubMed]

71. Sun, S. Multitask learning for EEG-based biometrics. In Proceedings of the 200819 th International Conference on Pattern Recognition, Tampa, FL, USA, 8-11 December 2008; pp. 1-4. [CrossRef]

72. Feinberg, I.; Braun, M.; Shulman, E. EEG sleep patterns in mental retardation. Electroencephalogr. Clin. Neurophysiol. 1969, 27, 128-141. [CrossRef]

73. Askaripoor, T.; Motamedzade, M.; Golmohammadi, R.; Farhadian, M.; Babamiri, M.; Samavati, M. Effects of light intervention on alertness and mental performance during the post-lunch dip: A multi-measure study. Ind. Health 2019, 57, 511-524. [CrossRef] [PubMed]

74. Wu, E.Q.; Deng, P.Y.; Qu, X.Y.; Tang, Z.; Zhang, W.M.; Zhu, L.M.; Ren, H.; Zhou, G.R.; Sheng, R.S. Detecting Fatigue Status of Pilots Based on Deep Learning Network Using EEG Signals. IEEE Trans. Cogn. Dev. Syst. 2020. [CrossRef]

75. Cartocci, G.; Maglione, A.G.; Vecchiato, G.; Di Flumeri, G.; Colosimo, A.; Scorpecci, A.; Marsella, P.; Giannantonio, S.; Malerba, P.; Borghini, G.; et al. Mental workload estimations in unilateral deafened children. Annu. Int. Conf. IEEE Eng. Med. Biol. Soc. 2015, 2015, 1654-1657. [CrossRef]

76. Duru, D.G.; Duru, A.D.; Barkana, D.E.; Sanli, O.; Ozkan, M. Assessment of surgeon's stress level and alertness using EEG during laparoscopic simple nephrectomy. In Proceedings of the 2013 6th International IEEE/EMBS Conference on Neural Engineering (NER), San Diego, CA, USA, 6-8 November 2013; pp. 452-455. [CrossRef]

77. Kalas, M.S.; Momin, B.F. Stress detection and reduction using EEG signals. In Proceedings of the 2016 International Conference on Electrical, Electronics, and Optimization Techniques (ICEEOT), Chennai, India, 3-5 March 2016; pp. 471-475. [CrossRef]

78. Lin, W.; Chen, Q.; Jiang, M.; Tao, J.; Liu, Z.; Zhang, X.; Wu, L.; Xu, S.; Kang, Y.; Zeng, Q. Sitting or Walking? Analyzing the Neural Emotional Indicators of Urban Green Space Behavior with Mobile EEG. J. Urban Health 2020, 97, 191-203. [CrossRef]

79. Anuragi, A.; Sisodia, D. Empirical wavelet transform based automated alcoholism detecting using EEG signal features. Biomed. Signal Process. Control 2019, 57, 101777. [CrossRef]

80. Liu, Y.; Sourina, O.; Nguyen, M.K. Real-Time EEG-Based Human Emotion Recognition and Visualization. In Proceedings of the 2010 International Conference on Cyberworlds, Singapore, 20-22 October 2010; pp. 262-269. [CrossRef]

81. Khushaba, R.; Wise, C.; Kodagoda, S.; Louviere, J.; Kahn, B.; Townsend, C. Consumer neuroscience: Assessing the brain response to marketing stimuli using electroencephalogram (EEG) and eye tracking. Expert Syst. Appl. 2013, 40, 3803-3812. [CrossRef]

82. Petukhov, I.V.; Glazyrin, A.E.; Gorokhov, A.V.; Steshina, L.A.; Tanryverdiev, I.O. Being present in a real or virtual world: A EEG study. Int. J. Med. Inform. 2020, 136, 103977. [CrossRef]

83. Vijayaragavan, G.R.; Raghav, R.L.; Phani, K.P.; Vaidyanathan, V. EEG monitored mind de-stressing smart phone application using Yoga and Music Therapy. In Proceedings of the 2015 International Conference on Green Computing and Internet of Things (ICGCIoT), Noida, India, 8-10 October 2015; pp. 412-415. [CrossRef] 
84. Mostow, J.; Chang, K.; Nelson, J. Toward Exploiting EEG Input in a Reading Tutor. In Artificial Intelligence in Education. AIED 2011. Lecture Notes in Computer Science; Biswas, G., Bull, S., Kay, J., Mitrovic, A., Eds.; Springer: Berlin/Heidelberg, Germany, 2011; Volume 6738.

85. Hsu, L.; Chen, Y. Neuromarketing, subliminal advertising, and hotel selection: An EEG study. Australas. Mark. J. 2020. [CrossRef]

86. Soria Morillo, L.; Alvarez-Garcia, J.; Gonzalez-Abril, L.; Ortega, J. Advertising Liking Recognition Technique Applied to Neuromarketing by Using Low-Cost EEG Headset; Springer: Cham, Switzerland, 2015; pp. 701-709. [CrossRef]

87. Gurgu, E.; Gurgu, I.; Bucea-Manea-Tonis, R. Neuromarketing for a better understanding of consumer needs and emotions. Indep. J. Manag. Prod. 2020, 11, 208. [CrossRef]

88. Li, S.; Cha, S.; Tappert, C.C. Biometric Distinctiveness of Brain Signals Based on EEG. In Proceedings of the 2018 IEEE 9th International Conference on Biometrics Theory, Applications and Systems (BTAS), Redondo Beach, CA, USA, 22-25 October 2018; pp. 1-6. [CrossRef]

89. la Rocca, D.; Campisi, P.; Scarano, G. EEG biometrics for individual recognition in resting state with closed eyes. In Proceedings of the 2012 BIOSIG-The International Conference of Biometrics Special Interest Group (BIOSIG), Darmstadt, Germany, 6-7 September 2012; pp. 1-12.

90. Wang, H.; Li, Y.; Hu, X.; Yang, Y.; Meng, Z.; Chang, K.-M. Using EEG to improve massive open online courses feedback interaction. In Proceedings of the CEUR Workshop Proceedings, Rio de Janeiro, Brazil, 3-4 April 2013; pp. 59-66.

91. Cirett Galan, F.; Beal, C. EEG Estimates of Engagement and Cognitive Workload Predict Math Problem Solving Outcomes. User Modeling, Adaptation, and Personalization; Springer: Berlin/Heidelberg, Germany, 2012. [CrossRef]

92. Frey, J.; Gervais, R.; Lainé, T.; Duluc, M.; Germain, H.; Fleck, S.; Lotte, F.; Hachet, M. Scientific Outreach with Teegi, a Tangible EEG Interface to Talk about Neurotechnologies; Association for Computing Machinery: New York, NY, USA, 2017. [CrossRef]

93. Mercier-Ganady, J.; Lotte, F.; Loup-Escande, E.; Marchal, M.; Lécuyer, A. The Mind-Mirror: See Your Brain in Action in your Head Using EEG and Augmented Reality. In Proceedings of the IEEE Virtual Reality, Minneapolis, MN, USA, 29 March-2 April 2014. [CrossRef]

Publisher's Note: MDPI stays neutral with regard to jurisdictional claims in published maps and institutional affiliations.

(C) 2020 by the authors. Licensee MDPI, Basel, Switzerland. This article is an open access article distributed under the terms and conditions of the Creative Commons Attribution (CC BY) license (http://creativecommons.org/licenses/by/4.0/). 